\title{
Structure reveals that BAK1 as a co-receptor recognizes the BRI1-bound brassinolide
}

Cell Research (2013) 23:1326-1329. doi:10.1038/cr.2013.131; published online 15 October 2013

\section{Dear Editor,}

In animals, steroid hormones play critical roles in many vital physiological processes. Brassinosteroids (BRs) produced by plants have similar structures to the animal steroid hormones and play indispensable roles in plant growth and development [1]. BRs have now acknowledgedly become a sixth class of plant hormones.

In contrast to animal steroid hormones that bind to nuclear receptors, BRs are recognized by the cell surface receptor BRASSINOSTEROID-INSENSITIVE 1 (BRI1) [2] and its homologs [3]. BRI1 belongs to a large family of plant leucine-rich repeat (LRR) receptor kinases (RKs), characterized by an extracellular ligand-sensing domain and an intracellular kinase domain. The extracellular domain of BRI1 contains 25 LRRs (BRI1LRR) and an island domain. Recognition of brassinolide (BL), the most active BR identified thus far, by BRI1LRR is through a surface pocket embedded in a 70-aa island (residues 587656) domain $[4,5]$. BR binding greatly enhances BRI1 heteromerization with a second RK, BRI1-associated kinase 1 (BAK1, also known as SOMATIC EMBRYOGENESIS RECEPTOR-LIKE KINASE 3, SERK3) [6, 7]. The BR-induced BRI1-BAK1 stable association initiates a phosphorylation-mediated cascade transducing the extracellular steroid signal to transcriptional programs $[1,3]$. This process is accompanied with dissociation of a BRI1 kinase inhibitor 1 (BKI1) from the intracellular domain of BRI1 [3]. Several models on BR-induced BRI1 activation have been proposed $[1,4,5,8]$. However, whether BAK1 acts as a regulator or co-receptor of BRI1 and how BR binding promotes BRI1-BAK1 interaction are not well understood.

To test whether the extracellular domains of BRI1 and BAK1 are sufficient to form a BL-induced complex, we expressed the LRR domains of Arabidopsis BRI1 (residues 24-784, BRI1LRR) and BAK1 (residues 1-220, BAK1LRR) in insect cells, purified the proteins to homogeneity, and tested their interaction using a gel filtration assay. Unexpectedly, when assayed with a buffer at $\mathrm{pH}$ 8.0, the BRI1LRR and BAK1LRR proteins did not form a stable complex (Figure 1A). As binding of BL to BRI1 occurs in the extracellular acidic space, we reasoned that lowering $\mathrm{pH}$ may favor formation of BLinduced BRI1LRR-BAK1LRR complexes. In complete agreement with the prediction, the two proteins strongly interacted with each other at $\mathrm{pH} 4.0$ in the presence of $\mathrm{BL}$, as indicated by co-migration of the two proteins in gel filtration, which was dependent on the existence of BL (Figure 1A). These results indicate that the extracellular domains of BRI1 and BAK1 interact with each other in a BL- and pH-dependent manner. The complex was eluted at the position corresponding to a monomeric BRI1LRR-BAK1LRR $(\sim 100 \mathrm{kD})$, suggesting that BL binding induces no homodimerization of the BRI1LRRBAK1LRR complex.

We then solved the crystal structure of the BRI1LRRBL-BAK1LRR complex at a resolution of $3.6 \AA$ (Supplementary information, Table S1). The BRI1LRRorganized complex is assembled with a 1:1:1 stoichiometry (Figure 1B). Supporting the gel filtration data, no homodimeric complexes were formed in the structure. Structural comparison showed that the conformations of BRI1LRR in the BAK1LRR-free and BAK1LRR-bound forms are markedly similar (Figure 1C), indicating that BAK1LRR binding induces no striking conformational changes in the extracellular domain of BRI1. As predicted from sequence alignment [6, 7], BAK1LRR contains five LRRs (Figure 1B). Its N-terminal capping domain can be well superimposed (Supplementary information, Figure S1) with those of BRI1LRR and BRL1LRR [9], supporting the idea that plant LRR proteins possess a conserved $\mathrm{N}$-terminal domain as suggested before [4].

Both BL and receptors mediate BRI1LRR-BAK1LRR heterodimerization. The N-terminal side of BAK1LRR makes contacts with the BRI1-bound BL (Figure 1B), resulting in the sandwich of BL between the two proteins and a nearly complete burial of the BL surface area $\left(\sim 4 \AA^{2}\right.$ after BAK1LRR binding). Contacts of the inner surface side of BAK1LRR with one lateral side of the last two LRRs from BRI1LRR form more extensive and direct BRI1LRR-BAK1LRR interactions. Ligand-induced 


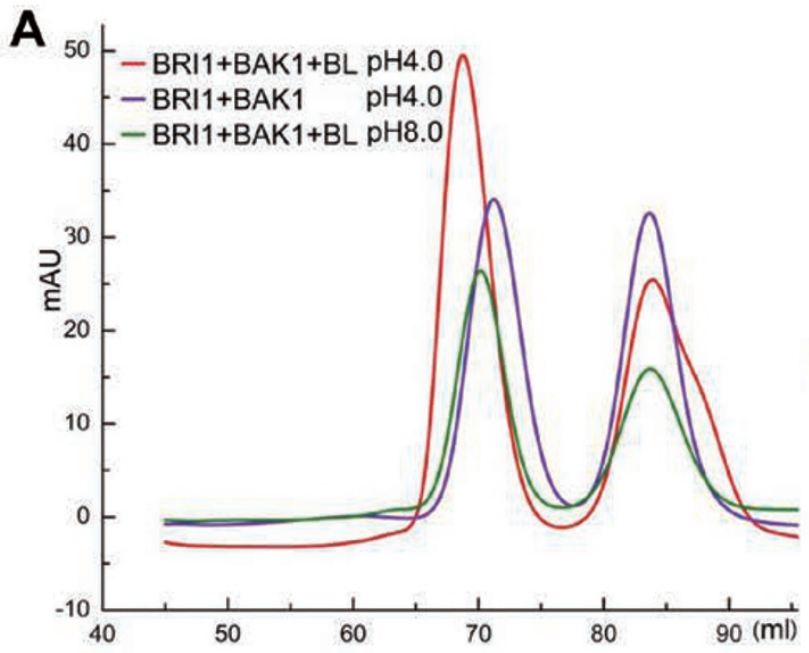

MM $68 \quad 69 \quad 71 \quad 73 \quad 75 \quad 76 \quad 83 \quad 84 \quad 85(\mathrm{ml})$
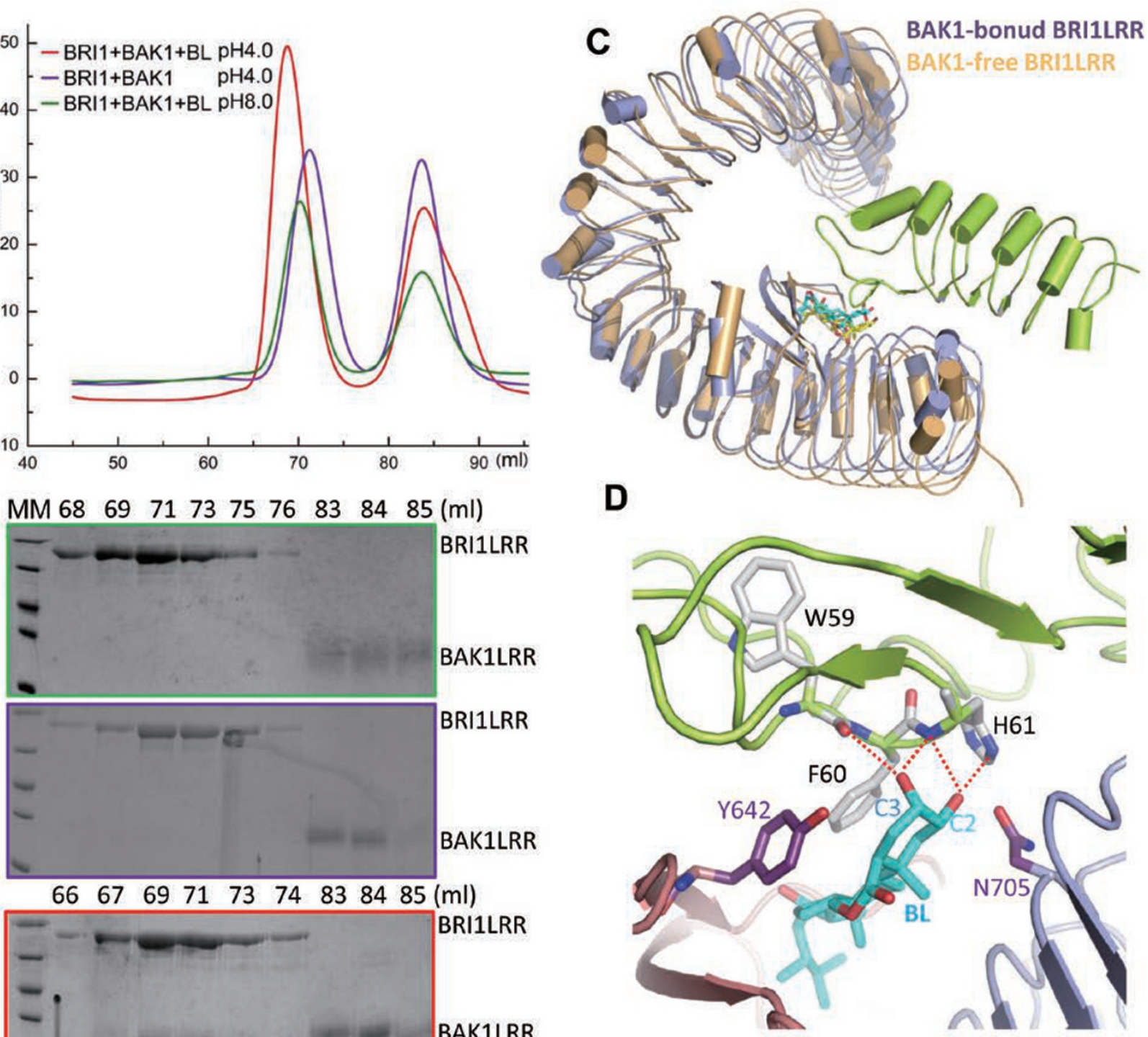

B
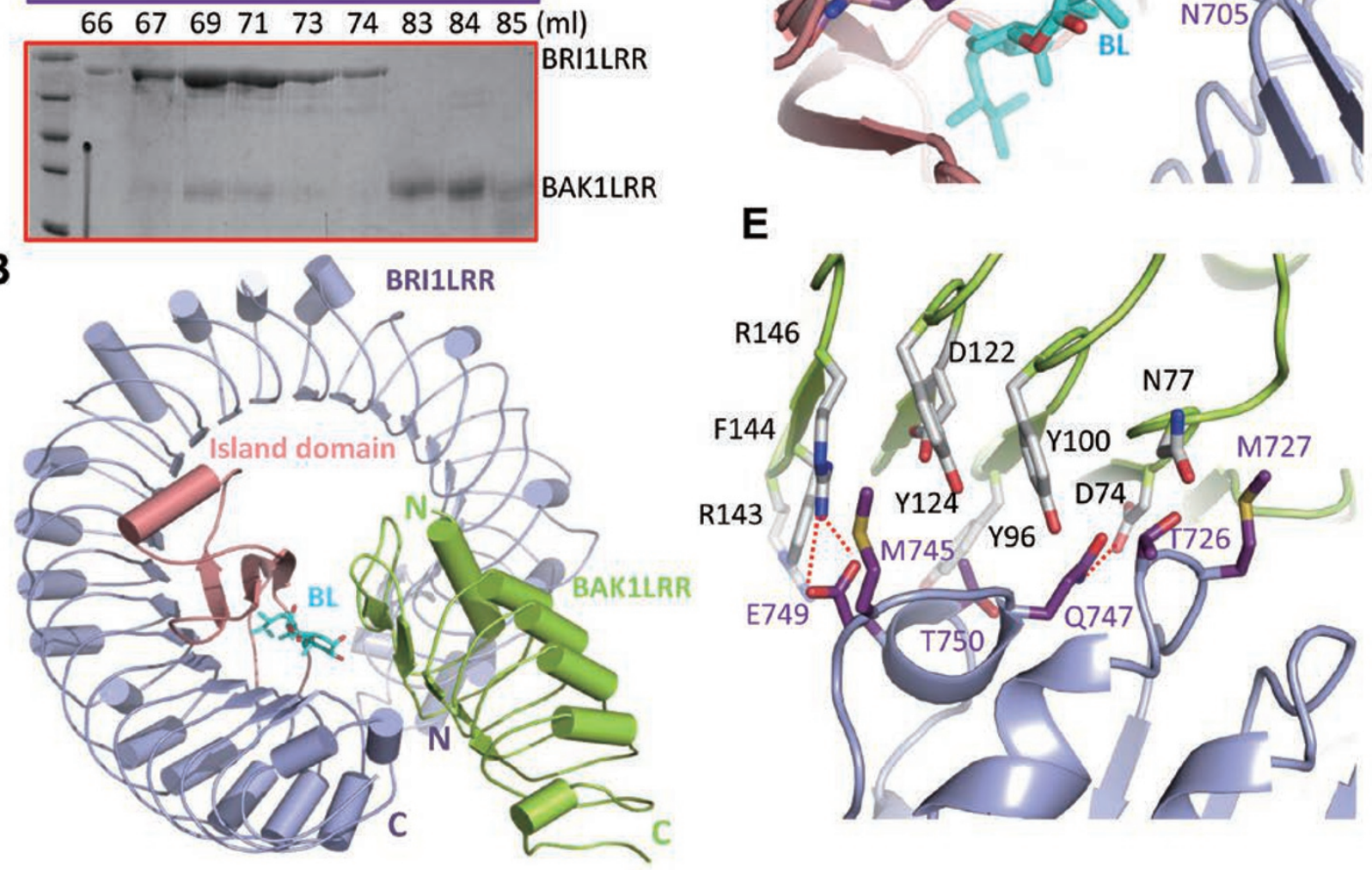
homo- or hetero-dimers of toll-like receptors (TLRs) typically have "m-shaped" structures [10]. The structural organization of the BRI1LRR-BAK1LRR dimeric complex is strikingly different from those of the TLR dimers. Nevertheless, the C-termini of BAK1LRR and BRI1LRR are similarly oriented (Figure 1B), presumably pointing to the membrane surface.

In the structure, BL was well defined by electron density (Supplementary information, Figure S2), interacting with BRI1LRR as observed before [4, 5]. In addition to BRI1LRR, BL also interacts with the $\mathrm{N}$-terminal side of BAK1LRR mainly through the fused ring portion, bridging BRI1LRR and BAK1LRR together (Figure 1B and Supplementary information, Figure S3). This structural observation supports our previous proposal [5] that BL induces BRI1-BAK1 interaction through creating a novel surface on BRILRR. BAK $1^{\text {Trp59, Phe60, His61 }}$ from the Nterminal capping domain are important for recognizing BL (Figure 1D). Specifically, the side chain of BAK1 $1^{\text {Phe60 }}$ tightly packs in parallel against one side of BL, substantially reducing the solvent accessible area of BL. Hydrogen bonding interactions of the three BAK1 residues with BL appear important for BAK1 recognition of the BRI1LRR-bound BL. The hydroxyl group from $\mathrm{C} 3$ of $\mathrm{BL}$ forms a bifurcated hydrogen bond with the carbonyl oxygen of BAK $1^{\text {Trp59 }}$ and the nitrogen of BAK $1^{\text {His61 }}$, whereas the hydroxyl group from $\mathrm{C} 2$ hydrogen bonds with the side chain and amide nitrogen of BAK $1^{\text {His61 }}$. In strong support of the important role for the hydrogen bonds in BL-induced BRI1LRR-BAK1LRR interaction, previous studies showed that modification either of the two hydroxyl groups greatly reduced the bioactivity of $\mathrm{BL}$ or its derivatives [11].

In addition to the BL-mediated interactions, BRI1LRR and BAK1LRR make extensive and direct contacts via packing of the inner surface of BAK1LRR against the last two LRRs of BRI1LRR (Figure 1B). A cluster of bulky amino acids including BAK1 1yr96, Tyr100, Tyr124, Arg143, Phe144, Arg146, establishes van der Waals and hydrophobic interactions with those from the C-terminal side of BRI1LRR (Figure $1 \mathrm{E})$. BRI $1^{\text {Thr750 }}$ is located at the center of the cluster and makes tight contacts with BAK $1^{\mathrm{Ty} r 96}$. Strained by the limited space around BRI ${ }^{\text {Thr750 }}$, substitution of this residue with a bulkier one is expected to perturb the direct BRI1LRR-BAK1LRR interactions. Indeed, the BRI1 ${ }^{\mathrm{T750}}$ mutation was shown to greatly compromise BL-induced signaling [12], providing functional evidence for our structural observation. In addition to the van der Waals and hydrophobic contacts, polar interactions also contribute to the direct BRI1LRR-BAK1LRR interface (Figure 1E). BAK $1^{\mathrm{Arg} 146}$ makes a pair of salt bridges with $\mathrm{BRI} 1^{\mathrm{G}-}$ ${ }^{14749}$, whereas the side chain of BAK $1^{\text {Asp } 74}$ hydrogen bonds to that of BRI $1^{\mathrm{Gln} 747}$. BAK $1^{\text {Asp122 }}$ is submerged among the BRI1-interacting cluster but makes no interaction with BRI1LRR. Mutation of this residue to asparagine, however, generates a gain-of-function phenotype [13]. The mechanism underlying this remains unclear, but glycosylation of the mutant protein at this position is unlikely responsible for the phenotype, because this would generate steric clashes with BRI1LRR. One possibility is that the mutation changed the local polarity and consequently the conformations of its surrounding BRI1-interacting residues, which in turn facilitates BRI1-BAK1 interaction. All the BL- and BRI1-interacting amino acids of BAK1 are highly conserved among other SERK members (Supplementary information, Figure S4).

In the current study, we solved the crystal structure of the BRI1LRR-BL-BAK1LRR complex, explaining a body of previous observations. The formation of the BLinduced BRI1LRR-BAK1LRR complex is stringently dependent on $\mathrm{pH}$, with lower $\mathrm{pH}$ promoting the BLinduced BRI1LRR-BAK1LRR interaction. Two possible mechanisms can be proposed to explain the $\mathrm{pH}$ dependent BRI1LRR-BAK1LRR heterodimerization induced by BL. Lowering $\mathrm{pH}$ may promote protonation of the amide nitrogen of BAK $1^{\text {His61 }}$ and allow it to act as two hydrogen bond donors for interaction with the two neighboring hydroxyl groups from the fused ring of BL.

Figure 1 BAK1LRR recognizes the BRI1LRR-bound $B L$ to form a pH-dependent BRI1LRR-BAK1LRR heterodimer. (A) BL induces BRI1LRR-BAK1LRR heterodimerization in solution at an acidic $\mathrm{pH}$. Top panel, gel filtration profiles of BRI1LRR and BAK1LRR in the presence (red at $\mathrm{pH} 4.0$ and green at $\mathrm{pH}$ 8.0) and absence (purple) of BL. The vertical and horizontal axes represent ultraviolet absorbance $(\lambda=280 \mathrm{~nm})$ and elution volume $(\mathrm{ml})$, respectively. BL, brassinolide. The $2 \mathrm{nd}, 3 \mathrm{rd}$ and 4 th panels are Coomassie blue staining of the peak fractions following SDS-PAGE. Frame colors indicate their corresponding gel filtration runs shown on the top. The numbers shown on top of the gels indicate elution volume $(\mathrm{ml})$. MM: molecular weight maker; the five bands from top to bottom indicate 112 kD, $66 \mathrm{kD}, 45 \mathrm{kD}, 35 \mathrm{kD}$ and $25 \mathrm{kD}$, respectively. (B) Overall structure of BRI1LRR-BL-BAK1LRR shown in cartoon. Color codes are indicated. "N" and "C" represent the $\mathrm{N}$ - and C-terminus, respectively. (C) BAK1LRR binding induces no striking conformational changes in BRI1LRR. Shown in the figure is structural comparison of the BAK1LRR-free and BAK1LRR-bound BLBRI1LRR. BL in BRI1LRR and BRI1LRR-BL-BAK1LRR is shown in yellow and cyan, respectively. (D) BAK1LRR recognizes the BRI1LRR-bound BL. Detailed interactions of BL with the N-terminal side of BAK1LRR. The side chains from BAK1LRR and BRI1LRR are shown in cream white and purple, respectively. Dashed red lines indicate hydrogen bonds. Two of the carbon atoms in $B L$ are labeled. (E) Direct interaction between BRI1LRR and BAK1LRR. Residues from BAK1LRR and BRI1LRR are labeled in black and purple, respectively. 
Alternatively or additionally, the $\mathrm{pH}$-dependent BL-induced BRI1LRR-BAK1LRR interaction may also be associated with conformations of the residues surrounding BAK $1^{\text {Asp } 122}$ that generates a gain-of-function phenotype when mutated to asparagine [13]. A basic $\mathrm{pH}$ might generate a different effect on conformations of those BAK1 residues from the BAK1 mutant, thus disallowing the BRI1LRR-BAK1LRR interaction for unknown reasons. Regardless of the mechanism underlying the $\mathrm{pH}$-dependent BRI1LRR-BAK1LRR interaction, our data suggest that a higher $\mathrm{pH}$ may be unfavorable for BR-induced signaling. The plant hormone auxin-dependent acidification of plant cell walls promotes wall extensibility and cell growth [14], whereas a hallmark of plant basal immunity is alkalinization (an increase in $\mathrm{pH}$ ) induced by phytopathogenic bacteria [15]. It will be interesting to investigate whether the alterations of $\mathrm{pH}$ accompanied with auxin treatment and plant immunity has an effect on BR-induced signaling.

Given that BAK1 has no BL-binding activity [8], their interactions observed in the structure should be dependent on binding of BL to BRI1LRR. Thus, the assembly of a signaling competent BRI1-BL-BAK1 complex is sequential with BL binding to BRI1 first followed by BAK1 specific recognition of the newly BL-created surface via hydrophobic and hydrogen bonding interactions. Connecting BRI1LRR and BAK1LRR by BL, at least partially, contributes to the BL-enhanced BRI1-BAK1 interactions. The hydrogen bonding interactions formed between BL and BAK1LRR may have a critical role in dictating their specific interaction. In keeping with this possibility, many BL derivatives that have a different fused ring portion but retain the two hydroxyl groups are bioactive [11]. Both the BL- and receptor-mediated BRI1LRR-BAK1LRR interactions appear important for signaling, as supported by the data derived from modifications of the two hydroxyl groups in BL and the BRI$1^{\text {T750I }}$ mutation. Therefore, we conclude that BAK1 acts a co-receptor of BRI1. Of note is that during review of our manuscript, a similar conclusion was made for SERK1 based on the crystal structure of BRI1LRR-SERK1LRR [16].

Detailed methods are described in the Supplementary information, Data S1.

\section{Acknowledgments}

We thank F Yu and J He at Shanghai Synchrotron Radiation Facility (SSRF). This work was funded by the State Key Program of National Natural Science of China (20111310576) and the National Outstanding Young Scholar Science Foundation of China (20101331722) to JC.

Yadong Sun ${ }^{1, *}$, Zhifu Han ${ }^{1, *}$, Jiao Tang ${ }^{1}$, Zehan $\mathrm{Hu}^{1}$, Chengliang Chai ${ }^{1}$, Bin Zhou ${ }^{1}$, Jijie Chai ${ }^{1}$

${ }^{I}$ Tsinghua-Peking Center for Life Sciences, Beijing 100084, China; School of Life Sciences, Tsinghua University, Beijing 100084, China

*These two authors contributed equally to this work.

Correspondence: Jijie Chai

E-mail: chaijj@mail.tsinghua.edu.cn

\section{References}

1 Clouse SD. Brassinosteroids. In: Torii K, Chang C, eds. The Arabidopsis Book. Plenum Press: The American Society of Plant Biologists. 2011:e151.

2 Li J, Chory J. Cell 1997; 90:929-938.

3 Jiang J, Zhang C, Wang X. J Integr Plant Biol 2013 May 29. doi: 10.1111/jipb.12081

4 Hothorn M, Belkhadir Y, Dreux M, et al. Nature 2011; 474:467-471.

5 She J, Han Z, Kim TW, et al. Nature 2011; 474:472-476.

6 Li J, Wen J, Lease KA, et al. Cell 2002; 110:213-222.

7 Nam KH, Li J. Cell 2002; 110:203-212.

8 Wang X, Li X, Meisenhelder J, et al. Dev Cell 2005; 8:855-865.

9 She J, Han Z, Zhou B, et al. Protein Cell 2013; 4:475-482.

10 Song DH, Lee JO. Immunol Rev 2012; 250:216-229.

11 Zullo MTA, Adam G. Braz J Plant Physiol 2002; 14:143-181.

12 Friedrichsen DM, Joazeiro CA, Li J, et al. Plant Physiol 2000; 123:1247-1256.

13 Jaillais Y, Belkhadir Y, Balsemao-Pires E, et al. Proc Natl Acad Sci USA 2011; 108:8503-8507.

14 Rayle DL, Cleland RE. Plant Physiol 1992; 99:1271-1274.

15 Felix G, Duran JD, Volko S, et al. Plant J 1999; 18:265-276.

16 Santiago J, Henzler C, Hothorn M. Science 2013; 341:889-892.

(Supplementary information is linked to the online version of the paper on the Cell Research website.)

(c) (i) () (2) This work is licensed under the Creative Commons Attribution-NonCommercial-ShareAlike 3.0 Unported License. To view a copy of this license, visit http://creativecommons.org/licenses/by-nc-sa/3.0 